\title{
Endodontia regenerativa em dentes permanentes com rizogênese incompleta
}

\author{
Regenerative endodontics in permanent teeth with incomplete rhizogenesis
}

Endodoncia regenerativa en dientes permanentes com rizogénesis incompleta

Daniela Pereira COSTA ${ }^{1}$

Letícia Neves ALMEIDA ${ }^{1}$

Letícia Reis AZEVEDO ${ }^{1}$

Jeane de Fátima Correia Silva ALVES ${ }^{2}$

${ }^{1}$ Graduação em Odontologia pelo centro Universitário Newton Paiva, 30431-262 Belo Horizonte -MG, Brasil

${ }^{2}$ Professora titular do curso de Odontologia do centro Universitário Newton Paiva, 30431-262 Belo Horizonte -MG, Brasil

\section{Resumo}

O tratamento endodôntico radical em dentes com ápices maduros, a apicificação em dentes com ápices imaturos e a exodontia são modalidades terapêuticas utilizadas nos casos de necrose pulpar. A endodontia regenerativa é uma alternativa para tratamento endodôntico de dentes com necrose pulpar e rizogênese incompleta. O presente estudo tem como objetivo analisar protocolos existentes e revisar as condutas clínicas associadas as terapias regenerativas, por meio de revisão da literatura do tipo descritiva. Foi realizada seleção de artigos científicos a partir das bases de dados Pubmed (National Center for Biotechnology Information) e Scielo (Scientific Eletronic Library Online) no período de 2014 a 2019. O principal benefício da técnica é possibilitar a reconstituição do tecido pulpar. A maioria dos autores empregam técnicas de revascularização pulpar. A utilização de métodos regenerativos foi observado em estudos in vitro e in vivo, utilizando modelos animais. Entretanto, muitos autores usam o termo endodontia regenerativa para se referir a revascularização. A revascularização é um procedimento regenerativo que permite a continuidade de desenvolvimento radicular. As técnicas de regeneração pulpar utilizam o conceito de engenharia tecidual para criar alternativas de restabelecer a vitalidade do elemento dentário. No entanto, muitas pesquisas são necessárias para avaliação e comparação de resultados obtidos, clinicamente, sobre essas novas terapias que envolvem os conceitos de endodontia regenerativa.

Descritores: Polpa Dentária; Raiz Dentária; Endodontia.

\section{Abstract}

Radical endodontic treatment in mature apex teeth, application to immature apex teeth and extraction are therapeutic used in cases of pulp necrosis. Regenerative endodontics is an alternative for endodontic treatment of teeth with incomplete pulp necrosis rhizogenesis. The present study aims to analyze existing protocols and review as associated clinical practices as regenerative therapies. This paper deals with a descriptive literature review. A selection of scientific articles was made from the Pubmed (National Center Biotechnology Information) and Scielo (Scientific Electronic Library Online) databases, and 47 articles were included in the present review. Among them, nine new cases of clinical cases were stand out about regenerative therapies printed from 2014 to 2019. The main benefit of the technique allows the pulp tissue to be reconstituted. Most authors employ pulp revascularization techniques. The use of regenerative methods has been observed in, in vitro and in vivo studies using animal models. However, many authors use the term regenerative endodontics to refer to revascularization. Revascularization is a regenerative procedure that allows the detection of root development. The pulp regeneration techniques use the concept of technological engineering to create alternatives to restore the vitality of the dental element. However, much research is needed to evaluate and compare clinical results with these therapie that involve the concepts of regenerative endodontics.

Descriptors: Dental Pulp; Tooth Root; Endodontics.

\section{Resumen}

El tratamiento de endodoncia radical en dientes con ápices maduros, la aplicación en dientes con ápices inmaduros y la extracción son modalidades terapéuticas utilizadas en los casos de necrosis pulpar. La endodoncia regenerativa es una alternativa para el tratamiento endodóntico de dientes con necrosis pulpar rizogénesis incompleta. El presente estudio tiene como objetivo analizar protocolos existentes y revisar las conductas clínicas asociadas a las terapias regenerativas. El trabajo trata de una revisión bibliográfica del tipo descriptiva. Fue realizada una selección de articulos científicos a partir de las bases de datos Pubmed (National Center Biotechnology Information) y Scielo (Scientific Eletronic Library Online) siendo incluídos 47 artículos en la presente revisión, entre ellos se destacan nueve relatos de casos clínicos sobre terapias regenerativas publicados entre los años 2014 y 2019. El principal beneficio de la técnica es posibilitar la reconstitución del tejido pulpar. La mayoria de los autores emplean técnicas de revascularización pulpar. La utilización de métodos regenerativos fue observado en estudos in vitro y in vivo, utilizando como modelo animales. Sin embargo, muchos autores usan el término endodoncia regenerativa para referirse a la revascularización. La revascularización es un procedimiento regenerativo que permite la continuidad del desenvolvimento radicular. Las técnicas de regeneración pulpar utilizan el concepto de engenieria tisular para crear alternativas restablecer la vitalidad del elemento dentario. Son necesarios estudios para evaluación y comparación de resultados obtenidos clinicamente sobre estas nuevas terapias que involucran los conceptos sobre endodoncia regenerativa.

Descriptores: Pulpa Dental; Raíz del Diente; Endodoncia.

INTRODUÇÃO

O tratamento endodôntico de dentes permanentes com necrose pulpar e rizogênese incompleta, é considerado um desafio na terapia endodôntica, pelo fato da necrose pulpar interromper o desenvolvimento radicular ${ }^{1}$.

A polpa dentária é constituída por um tecido conjuntivo frouxo envolvido por tecido dentinário. Os odontoblastos, células presentes na superfície pulpar é responsável pela formação da dentina. Histologicamente a polpa dentária é dividida em camadas: (1) Camada odontoblástica na periferia; (2) Camada subodontoblástica com as zonas pobres em células e zona ricas em células; (3) Região central da polpa caracterizada por vasos e nervos maiores da polpa ${ }^{2}$. Logo abaixo dos odontoblastos, encontra-se uma camada de células indiferenciadas e células de defesa pulpar. Os fibroblastos são responsáveis pela formação de fibras colágenas e proteínas da matriz do tecido pulpar. Os linfócitos $T$ participam da imunidade celular, e os linfócitos 
B e plasmócitos, participam da síntese de anticorpos, não comum em polpa normal. Os macrófagos fagocitam restos celulares e substâncias irritantes a polpa, e também como apresentadores de antígenos ${ }^{3}$.

A regeneração endodôntica pode ser definida como uma terapia promissora com base biológica que visa repor estruturas pulpares danificadas. As tecnologias de engenharia de tecidos fornecem novos meios de potenciais para a construção de uma "polpa artificial", projetados para substituir as estruturas pulpares danificadas ${ }^{4}$.

A revascularização é um procedimento que através da indução de sangramento na região periapical, promove a formação de um coágulo sanguíneo, com presença de célulastronco, que estimulam a formação de um novo tecido ${ }^{5}$.

Atualmente, a apicificação é um procedimento realizado para promover a formação de uma barreira apical artificial em dentes imaturos com necrose pulpar, e assim permitir a inserção do material obturador. A desvantagem dessa técnica é impedir o contínuo desenvolvimento radicular em termos de maturação apical e espessamento das paredes dentinárias tornando 0 elemento dentário mais frágil e susceptível a fratura ${ }^{6}$.

As pesquisas atuais sobre terapias regenerativas e de revascularizações no meio da endodontia buscam estabelecer um tratamento mais eficaz, viável e seguro do que as terapias pulpares tradicionais como a apicificação para dentes com rigozênese incompleta e necrose pulpar ${ }^{7,8}$.

Segundo Nosrat et al. $^{9}$ a terapia regenerativa, apresentaria uma grande probabilidade de sucesso pois permite à revitalização da polpa necrosada, 0 que permitiria a continuação do crescimento radicular, o fechamento do forame apical e o aumento da espessura dentinária em dentes imaturos.

Apesar da terapia regenerativa e de revascularização serem promissoras, apresenta-se poucos estudos realizados até o atual momento. Além disso, muitos protocolos clínicos na literatura foram descritos, e não há um consenso entre os autores sobre a técnica mais indicada ${ }^{10}$. Sendo assim, o objetivo do presente estudo foi realizar uma revisão de literatura sobre as terapias regenerativas e de revascularização pulpar.

MATERIAL E MÉTODO

Realizou-se uma revisão da literatura onde foram analisados artigos científicos obtidos da base de dados PubMed (National Center for Biotechnology Information) e Scielo (Scientific Eletronic Library Online). Foram utilizadas as palavras-chave: Revascularização Pulpar; Regeneração Pulpar; Endodontia Regenerativa, bem como a associação dos termos pesquisados nos idiomas inglês, espanhol e português. Foram incluídos 34 artigos na presente revisão, dentre eles destacam-se nove relatos de casos clínicos sobre terapias regenerativas publicados entre os anos de 2014 a 2019. Os dados encontrados destes artigos estão descritos nos quadros um e dois.

\section{RESULTADOS}

- Revisão da literatura

Os dentes permanentes com rizogênese incompleta são aqueles que não apresentam o ápice e a raiz totalmente formados. A apicificação é a técnica atual realizada em dentes imaturos com necrose pulpar, por meio da colocação do hidróxido de cálcio com a finalidade de formar uma barreira apical artificial para realizar a obturação. Após a terapia endodôntica foi observada histologicamente a presença de um novo tecido, levando a crença de que a regeneração era possível. Contudo, o dente após a terapia é mais susceptível à fratura, devido à presença de finas paredes dentinárias, que não se espessaram devido à interrupção do desenvolvimento radicular ${ }^{11}$. Além disso, a permanência do hidróxido de cálcio em longos períodos de tempo no canal radicular pode provocar a fratura da raiz devido as suas propriedades higroscópicas e proteolíticas $^{12}$.

A revascularização consiste na desinfecção dos canais radiculares, seguida de uma indução de sangramento na região periapical, o que promove o preenchimento do canal com a formação de um coagulo sanguíneo e células indiferenciadas ${ }^{13-17}$. Segundo Shan et al. ${ }^{18}$, estas células estimulam a formação de um novo tecido pulpar. No Quadro 1 estão descritos os casos clínicos de revascularização no período de 2014 a 2019.

A regeneração pulpar é definida como uma terapia que promove a diferenciação de células progenitoras da porção apical de dentes jovens, permitindo assim a deposição de tecidos mineralizados nas paredes dentinárias ${ }^{1,6,19-21}$. No Quadro 2 estão descritos os protocolos clínicos de regeneração no período de 2014 a 2019.

A revascularização e a regeneração são procedimentos regenerativos promissores que tem a finalidade de permitir o contínuo 
desenvolvimento radicular em dentes imaturos com necrose pulpar ${ }^{22}$.

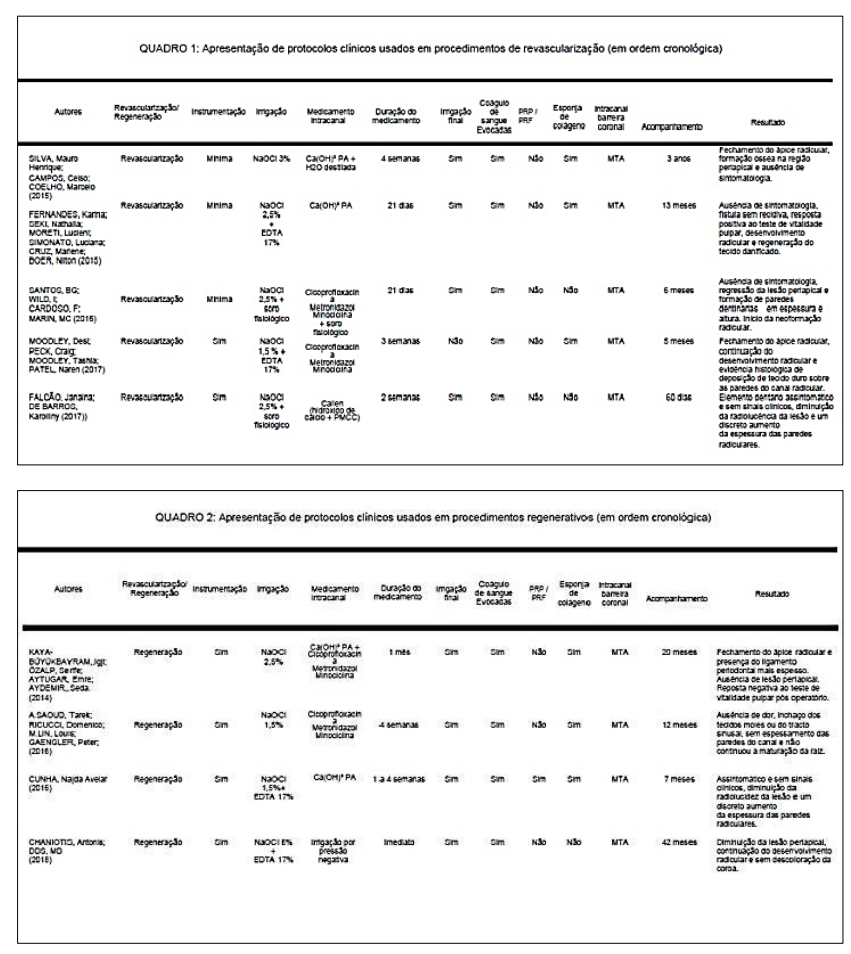

A instrumentação baseia-se em conjuntos de limas que são utilizadas para dar alargamento, modelagem e acabamento ao canal $^{23}$. No tratamento de dentes com rizogênese incompleta 0 uso de limas para instrumentação endodôntica é evitado ou reduzido para que o elemento dentário não se torne ainda mais frágil ${ }^{13,14,17,24}$.

As soluções irrigadoras são essenciais para a desinfeção primária tendo que possuir máximo efeito bactericida e bacteriostático e mínimo efeito citotóxico sobre as células estaminais e sobre os fibroblastos, para que possam sobreviver e continuar com a capacidade de proliferação. Sendo assim a escolha correta das soluções irrigadoras é crucial para o sucesso da terapia regenerativa ${ }^{5}$.

O hipoclorito de sódio ( $\mathrm{NaOCl}$ ) é uma solução irrigadora que pode ser utilizado nas concentrações de $0,5 \%$ a $6 \%$, sendo a mais indicada pelos autores para a desinfecção dos canais radiculares, devido à sua capacidade de dissolução tecidual e amplo espectro de ação antimicrobiana contra os principais patógenos endodônticos ${ }^{13-27,19,20,21,26}$.

O EDTA é uma substância fluída com um pH neutro de 7,3 utilizado na concentração de $10 \%$ a $17 \%$. É um agente quelante capaz de remover smear layer, que é um material com aparência amorfa, irregular e granular, formada nas paredes dentinárias ${ }^{13,15,20,26,27}$. A solução fisiológica tem um efeito neutralizador, que diminui a citoroxicidade aos remanescentes celulares na região periapical, e evita a interação das moléculas de hipoclorito de sódio com as de clorexidina, quando utilizadas juntas no tratamento pulpar ${ }^{14,16,28}$.

Segundo Albuquerque et al..$^{22}$, uma das razões de sucesso da regeneração pulpar é a eliminação de microrganismos. Por isso, a medicação intracanal apresentar um papel fundamental na sanificação dos canais radiculares, pelo processo de revascularização pulpar ser mais favorável em ambientes livres de bactérias.

A pasta tri-antibiótica é uma medicação intracanal importante para o controle de infecção no interior dos sistemas de canais radiculares, por possibilitar a criação de condições ambientais nos sistemas de canais radiculares para a proliferação de um novo tecido pulpar capaz de dar continuar ao desenvolvimento radicular. A pasta é composta por $400 \mathrm{mg}$ de metronidazol, $250 \mathrm{mg}$ de ciprofloxacina e $50 \mathrm{mg}$ de minociclina, manipulada em propilenoglicol ${ }^{14,15,19,21}$.

A eficácia do hidróxido de cálcio sobre microrganismos e tecidos está diretamente relacionada com a sua dissociação iônica em íons de cálcio e hidroxila. A sua ação é devido ao seu alto $\mathrm{pH}$ que inibe a atividade de enzimas que são essenciais para a vida bacteriana $^{3,13,16,17,20,21}$.

O Agregado Trióxido Mineral (MTA) tem como vantagens a ausência ou a baixa resposta inflamatória tecidual, menor infiltração, além de apresentar características antimicrobianas devido ao seu $\mathrm{pH}$ básico. Esse cimento também se destaca por ser de fácil manipulação, por favorecer a formação de tecido duro e possuir baixa solubilidade. Porém apresenta algumas desvantagens como longo tempo de presa, baixa resistência à compressão e um alto custo $^{13-17,19-21,26,29}$.

- Técnica atualmente em estudo de revascularização endodôntica

- Revascularização do canal radicular através de um coágulo de sangue

A revascularização de dentes necrosados com rizogênese incompleta tem como finalidade reparo da lesão periapical, eliminação de sintomas, continuação do desenvolvimento radicular e reestabelecimento da vitalidade pulpar ${ }^{4}$. O protocolo geralmente é realizado em duas consultas. $\mathrm{Na}$ primeira realiza-se a limpeza do sistema de canais radiculares por meio de irrigação e inserção de medicação intracanal, que deve permanecer aproximadamente 3 semanas. $\mathrm{Na}$ segunda sessão é feita a evocação do coágulo sanguíneo, seguida do selamento com MTA e compostos resinosos ${ }^{10,13-17}$. 
A AAE (American Association of Endodontics) elaborou no ano de 2013 um documento intitulado "Considerations for Regenerative Procedures", o qual descreve algumas recomendações sobre a técnica de revascularização com o objetivo de ajudar o clínico. No entanto, ainda não existe consenso quanto ao protocolo que se deve seguir nesse tipo de abordagem terapêutica ${ }^{4}$.

De acordo com a AAE, na primeira consulta é realizada anestesia local, seguida de isolamento absoluto com dique de borracha e cavidade de acesso. Na sequência é realizada irrigação abundante com $20 \mathrm{~mL}$ de $\mathrm{NaOCl}$. Após a secagem dos canais com pontas de papel, é colocada uma pasta triantibiótica com mistura $1: 1: 1$ de ciprofloxacino, metronidazol e minociclina, associada a uma pasta de hidróxido de cálcio, sendo selado com material restaurador temporário ${ }^{4}$.

Uma a quatro semanas após a primeira consulta é realizada anestesia com mepivacaína $3 \%$ sem vasoconstritor e colocado um isolamento absoluto. Após é feito uma irrigação abundante com $20 \mathrm{~mL}$ de EDTA $17 \%$ e secagem dos canais com pontas de papel, para evocação do coágulo sanguíneo por meio de instrumentação, e assim inserir um material restaurador. Se necessário, colocar uma matriz sobre o coágulo sanguíneo de MTA branco ou hidróxido de cálcio como material de capeamento, e acima uma camada de 3 - $4 \mathrm{~mm}$ de ionômero de vidro e uma restauração definitiva adesiva com compósito ${ }^{4}$.

\section{- Pespectivas futuras sobre terapias regenerativas em Endodontia}

- Terapia de células estaminais

Uma célula estaminal ou tronco é definida como uma célula que tem capacidade de dividir e produzir células descendentes que se diferenciam em vários outros tipos de células e tecidos ${ }^{30}$.

Até o presente momento, cinco tipos de células estaminais dentárias foram isolados e caracterizados: (1) Células estaminais da polpa dentária (DPSCs); (2) Células estaminais de dentes decíduos (SHED); (3) Células estaminais da papila apical (SCAP); (4) Células estaminais do ligamento periodontal (PDLSCs); (5) Células do folículo dentário (DFPCs). Todas estas células estaminais demonstram multipotencialidade e capacidade para regenerar múltiplos tecidos dentários e periodontais in vitro e in vivo ${ }^{6}$.

Entre os vários tipos de células que foram contemplados, as DPSCs da polpa são consideradas as células lógicas de escolha devido suas propriedades de auto-renovação e diferenciação, e suas vantagens associadas à sua aplicação em engenharia de tecidos, como o fácil acesso ao local de coleta destas células e baixa mobilidade. Sua extração do tecido pulpar é altamente eficiente, pela capacidade de diferenciação extensiva e boa interatividade com os biomateriais. A fácil gestão das DPSCs faz delas células viáveis e promissoras para o uso futuro em ensaios clínicos humanos ${ }^{4}$.

\section{- Implantação pulpar}

Nessa técnica, o tecido pulpar de substituição é transplantado em canais radiculares limpos e formatados ${ }^{7}$. A fonte de tecido pulpar pode ser uma linhagem de células estaminais purificadas isentas de agentes patogénicos. Também podem ser criadas a partir de uma biópsia do paciente para remoção celular. Logo após as células são cultivadas em laboratório e são induzidas a se diferenciarem em tecido pulpar. A cultura de células pulpares é realizada in vitro, utilizando polímeros biodegradáveis de nanofibras ou lâminas de proteínas da matriz extracelular, como o colágeno tipo I ou fibronectina ${ }^{31}$.

- Implantação de um scaffold

Scaffolds são estruturas rígidas e tridimensionais preparadas para fornecer suporte e organização das células estaminais, para que se torne possível a vascularização celular. Isso pode ser obtido através de um polímero poroso que contém fatores de crescimento e nutrientes para auxiliar na proliferação e diferenciação celular. O scaffold também pode conter antibióticos para prevenir qualquer crescimento bacteriano nos canais radiculares ${ }^{32}$.

Os materiais utilizados na síntese de scaffold podem ser naturais como colágeno, dentina, fibrina, seda e alginato, podendo se apresentar também em formas sintéticas como os poliésteres que se degradam no interior do corpo humano. O tipo de scaffold natural mais estudado é o de colágeno. O tipo de scaffold sintético mais utilizado é o de polímeros lático e glicólico ${ }^{7}$.

A técnica de scaffold injetável, consiste na colocação de um polímero de hidrogel nos canais radiculares que possui propriedades físicas similares aos tecidos vivos, potencial de não serem invasivos e de fácil aplicação nos sistemas de canais radiculares ${ }^{7}$.

O fosfato $\beta$-tricálcico, é um scaffold injetável em estudo que se trata de um alginato em fase de gel que formam gotas. A matriz de dentina tratada promove um ambiente favorável para a regeneração dos tecidos dentários ${ }^{4}$. Os scaffolds de seda podem ser utilizados, uma vez que o tamanho e a forma dos poros podem 
guiar a formação de tecidos mineralizados. Derivados da matriz de esmalte, composta principalmente por amelogeninas, também pode ser utilizada ${ }^{30}$.

\section{- Impressão celular tridimensional}

$\mathrm{Na}$ teoria, a técnica de impressão celular tridimensional utiliza um jato de tinta, que dispõe camadas de células suspensas em hidrogel para recriar a estrutura do tecido pulpar, sendo também utilizada para posicionar as células em uma posição precisa ${ }^{4}$. A posição ideal de células em uma construção de tecidos, passa pela colocação de células odontoblásticas em torno da periferia que servem para a manutenção e reparação da dentina. Com os fibroblastos na região central da polpa dentária para apoiar uma rede de células vasculares e nervosas $4,7,32$.

\section{- Terapia Genética}

Técnicas envolvendo vetores virais podem alterar a sequência genética de genes que codificam fatores de crescimento, fatores de transcrição e proteínas da matriz extracelular de determinadas populações celulares ${ }^{7}$.

$\mathrm{Na}$ abordagem in vivo o vetor viral é injetado na corrente sanguínea ou localmente no tecido alvo. Este método aumenta o potencial de regeneração do tecido pulpar, com a utilização de genes indutores da diferenciação de odontoblastos, com subsequente deposição de dentina que são aplicados diretamente na polpa exposta ${ }^{7,30}$.

O Plasma Rico em Fibrina (PRF) é um subproduto obtido do Plasma Rico em Plaquetas que foi desenvolvido para intensificar o aceleramento da reparação de tecidos ósseos e moles. A Fibrina Rica em Plaquetas e Leucócitos (L-PRF) é um material rico em plaquetas autólogas, fatores de crescimentos, e que apresentam um concentrado imunológico e plaquetário que possibilita a osteocondução e intensificam a resposta regenerativa das células do próprio paciente ${ }^{20}$.

DISCUSSÃO

Com o uso das técnicas regenerativas, os riscos de fraturas dentárias podem reduzir devido ao fato das mesmas possibilitarem o continuo desenvolvimento da raiz, o aumento de espessura das paredes dentinárias e 0 fechamento do ápice radicular. Além disso, a técnica proporciona a diminuição do tempo de tratamento por não ser necessário trocas periódicas de medicações intracanais, melhorando assim o prognóstico dentário ${ }^{13-17}$.

Nos protocolos clínicos encontrados, a maioria dos autores empregam a técnica de revascularização para ocorrer a revitalização pulpar $^{13-17,19,21,26}$. No entanto, alguns autores denominam a técnica de regeneração, e executam protocolo de revascularização ${ }^{15,16,19-21,26}$. Porém não está claro na literatura se o tecido regenerado na técnica de revascularização apresenta a mesma constituição histológica da polpa dentária. Alguns relatos publicados evidenciam a contínua formação da raiz e o elemento dentário passa a apresentar resposta positiva aos testes de sensibilidade pulpar. Apesar da AAE ter publicado um protocolo de técnica de revascularização, observa-se na literatura uma diversidade de métodos para execução do procedimento, o que torna difícil comparar os resultados existentes ${ }^{13-17,19-21,26}$.

Entre as técnicas encontradas de regeneração, a terapia de células estaminais, é uma técnica possível de ser realizada em consultório odontológico ${ }^{4,6,10}$. Essas células são coletadas do próprio paciente, o que torna o procedimento de baixo custo, e não ser necessário enfrentar processos éticos e legais. As células estaminais dentárias mais promissoras para o uso na regeneração pulpar são as DPSCs devido a sua facilidade de coleta e por apresentar as propriedades de autorenovação e capacidade de se diferenciar em outros tipos celulares. Já a técnica de implantação pulpar, até o momento não foi realizado em humano. Foi observado no estudo in vitro, que essa técnica apresenta a vantagem de agregar células e possibilitar a fixação das células implantadas em uma localização específica dentro do sistema de canais radiculares. Porém esse tecido pulpar apresenta o mesmo diâmetro do canal radicular o que dificulta a sua implantação. Outro método promissor utilizado in vitro para a fixação de células estaminais consiste na inserção de scaffold $^{32}$. Porém são necessários novos estudos para encontrar um scaffold adequado que permita a vascularização tecidual.

Os únicos autores que descreveram técnicas de regeneração pulpar em humanos foram Torabinejad et al. $^{33}$, Murray et al. $^{7}$ e Bansal et al. ${ }^{30}$. Os casos não foram descritos no quadro por não pertencerem ao período de 2014 a 2019. O primeiro realizou a técnica de scaffold injetável com o uso de PRP em um rapaz de 11 anos e observou fechamento apical e regressão da lesão periapical ${ }^{10}$. O segundo e o terceiro realizaram terapia genética para regeneração pulpar de dentes com rizogênese incompleta em pacientes com doenças terminais $^{7,30}$. A terapia genética emprega a modificação de genes em fatores de crescimento, morfogênese, fatores de 
transcrição de células alvo por meio de vetores virais ou não virais modificados. As alterações de morfogêneses podem estimular a formação de dentina reparadora. No entanto, foi observado o aparecimento de tumores em diferentes partes do corpo de um rapaz de 9 anos, submetido a essa técnica, o que levou a abolição da terapia regenerativa. A maioria dos métodos regenerativos utilizados foram descritos in vitro e alguns in vivo em animais, com isso é necessário novos estudos e pesquisas acerca das técnicas regenerativas para que sejam métodos confiáveis, viáveis e que apresentem bons resultados clinicamente.

Alguns dos protocolos de endodontia regenerativa revisados no quadro, descrevem o uso de instrumentação endodôntica para realizar a limpeza dos canais radiculares ${ }^{15,16,19-21,26}$. Entretanto, outros autores utilizaram o mínimo de instrumentação e obtiveram resultados satisfatórios como nos casos em que foram realizados instrumentação completa no canal radicular24,25,32. A evocação do coágulo sanguíneo por meio de instrumentos endodônticos levados a região periapical foram realizados por todos os autores com o intuito de que ocorra a revitalização pulpar ${ }^{13-17,19-21,26,30}$. A formação do coágulo sanguíneo parece ser importante para 0 sucesso da terapia regenerativa.

Todos os autores utilizaram como solução irrigadora nos canais radiculares o hipoclorito de sódio sozinho ou associado com outras soluções, devido às suas propriedades antimicrobianas $^{13-17,19-21,26,30}$. O uso dessa solução isolada ou associada à outra não interferiu nos resultados clínicos encontrados.

Alguns pesquisadores, escolheram a pasta tri-antibiótica como medicação intracanal, para tornar possível os processos de regeneração e revascularização ${ }^{14,15,19,21}$. Outros autores escolheram a pasta de hidróxido de cálcio como alternativa para medicação intracanal nos protocolos regenerativos ${ }^{13,16,17,21,22}$. Os resultados clínicos descritospelos autores foram semelhantes.

Cunha $^{20}$ utilizou o plasma rico em plaquetas e fibrinas como material para auxiliar na formação da raiz radicular e das paredes dentinárias. Entretanto não houve redução no tempo para o fechamento do ápice radicular e o espessamento de paredes dentinárias no caso clinico em que utilizou PRP em comparação com os que não utilizaram ${ }^{13,14,16,17,19,20,21,26}$.

Os autores foram unanimes em relação

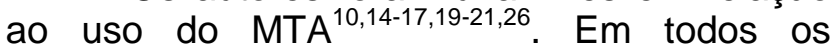
trabalhos revisados foi o material empregado como barreira coronal intracanal, devido ao fato de ser biocompatível com os tecidos pulpares, por auxiliar na remoção de microrganismos e ser resistente a infiltrações.

CONCLUSÃO

$\begin{array}{ccc}\text { Através } & \text { dessa } & \text { revisão foram } \\ \text { encontrados trabalhos } & \text { promissores que }\end{array}$
sinalizam a importância do emprego das técnicas de endodontia regenerativa para o tratamento de dentes com diagnóstico de necrose pulpar e rizogênese incompleta. Entretanto, a maioria dos autores empregam técnicas de revascularização pulpar. As utilizações de métodos regenerativos foram descritos in vitro e alguns in vivo em animais. Novos estudos e pesquisas acerca das técnicas regenerativas são necessárias. Além disso, torna-se necessário o estabelecimento de um protocolo padrão viável que possa ser seguido para execução das técnicas com segurança no consultório odontológico e que apresente um bom prognóstico. Dessa forma, estudos a longo tempo poderão ser realizados para comparar os resultados obtidos clinicamente.

\section{REFERÊNCIAS}

1. Resende EF. Regeneração pulpar como alternativa para tratamento endodôntico em dentes permanentes imaturos - revisão de literatura [monografia]. Belo Horizonte: Faculdade de Odontologia da Universidade Federal de Minas Gerais - UFMG; 2016.

2. Ten Cate NA. Histologia oral: desenvolvimento, estrutura e função. Rio de Janeiro: Elsevier; 2008.

3. Estrela C. Ciência Endodôntica. São Paulo: Artes Médicas, 2007.

4. Torres JCM. Técnica de regeneração endodôntica. [dissertação] Porto: Universidade Fernando Pessoa; 2011.

5. Namour M, Theys S. Pulp revascularization of immature permanent teeth: a review of the literature and a proposal of a new clinical protocol. Scientific World Journal. 2014;2014:737503.

6. Huang GT. Pulp and dentin tissue engineering and regeneration: current progress. Regen Med. 2009;4(5):697-707.

7. Murray PE, Garcia-Godoy F, Hargreaves KM. Regenerative endodontics: a review of current status and a call for action. J Endod. 2007;33(4):377-90.

8. Nakashima $M$. Tissue engineering in endodontics. Aust Endod J. 2005;31(3):111-13.

9. Nosrat A, Seifi A, Asgary S. Regenerative endodontic treatment (revascularization) for necrotic immature permanent molars: a review and report of two cases with a new biomaterial. J Endod. 2011;37(4):562-67.

10. Alcade MP, Guimarães BM, Fernandes SL, 
Amorosos-Silva PA, Bramante CM, Ricci R et al. Revascularização pulpar: considerações técnicas e implicações clínicas.Saluvista. 2014;33(3:415-32.

11. Witherspoon DE, Small JC, Regan JD, Nunn M. Retrospective analysis of open apex teeth obturated with mineral trioxide aggregate. J Endod. 2008;34(10):1171-76.

12. Andreasen JO, Munksgaard EC, Bakland LK. Comparison of fracture resistance in root canals of immature sheep teeth after filling with calcium hydroxide or MTA. Dent Traumatol. 2006;22(3):154-56.

13. Fernandes K, Seki N, Moreti L, Simonato L, Cruz M, Boer N. Regeneração endodôntiva em dente permanente jovem portador de necrose pulpar e rizogênese incompleta: relato de caso. Arch Health Invest. 2017;6(7):338-42.

14. Santos BG, Wild I, Cardoso FGR, Marin Mcc. Revascularização pulpar: relato de caso clínica. $X X$ Encontro Latino Americano de Iniciação Científica, XVI Encontro Latino Americano de Pós-Graduação e VI Encontro de Iniciação à Docência - Universidade do Vale do Paraíba. p.1-6

15. Moodley DS, Peck C., Moodley T, Patel N. Management of necrotic pulp of immature permanent incisor tooth: A regenerative endodontic treatment protocol: case report. S Afr dent j. 2017;72(3):122-25.

16. Falcão JMS, Barros KST. Revascularização pulpar em dente traumatizado: relato de caso clínico. Recife: Faculdade Integrada de Pernambuco - FACIPE; 2017.

17. Silva MH, Campos CN, Coelho MS. Revascularization of an Immature Tooth with Apical Periodontitis Using Calcium Hydroxide: A 3-year Follow-up. Open Dent J. 2015;9:482-85.

18. Shah N, Logani A, Bhaskar U, Aggarwal V. Efficacy of revascularization to induce apexification/apexogensis in infected, nonvital, immature teeth: a pilot clinical study. J Endod. 2008;34(8):919-25; Discussion 1157.

19. Saoud TMA, Ricucci D, Lin LM, Gaengler P. Regeneration and Repair in Endodontics-A Special Issue of the Regenerative EndodonticsA New Era in Clinical Endodontics. Dent $\mathrm{J}$ (Basel). 2016;4(1):3.

20. Cunha NA. Regeneração pulpar pós-trauma: relato de caso clínico. Belo Horizonte: Faculdade de Odontologia da Universidade Federal de Minas Gerais - UFMG; 2016.

21. Kaya-Büyükbayram I, Özalp Ş, Aytugar E, Aydemir S. Regenerative endodontic treatment of an infected immature dens invaginatus with the aid of cone-beam computed tomography. Case Rep Dent. 2014;2014:403045.

22. Albuquerque MTP, Nagata JY, Soares AJ, Zaia AA. Pulp revascularization: alternative treatment to the apexification of immature teeth. Rev Gaúch de Odontolol. 2014;62(4):401-10.

23. Souza TS, Deonízio MA, Batista A, Kowalczuck A, Sydney GB. Regeneração endodôntica: existe um protocolo? Rev Odontol Bras Central. 2013; 22(63):128-33.

24. Nascimento RAS, Santana RM. Revascularização endodôntica. Repositório da Uberaba: Universidade de Uberaba - UNIUBE; 2018.

25. Ferraz CCR, Gomes BPFA, Zaia AA, Teixeira FB, Souza-Filho FJ.Comparative study of the antimicrobial ef-ficacy of chlorhexidine gel, chlorhexidine solution and sodium hypochlorite as endodontics irrigants. Braz Dent J. 2007;18(4):294-98.

26. Chaniotis A. Orthodontic Movement after Regenerative Endodontic Procedure: Case Report and Long-term Observations. J Endod. 2018;44(3):432-37.

27. Sen $B H$, Wesselink $P R$, Türkün $M$. The smear layer: a phenomenon in root canal therapy. Int Endod J. 1995;28(3):141-48.

28. Reynolds K, Johnson JD, Cohenca N. Pulp revascularization of necrotic bilateral bicuspids using a modified novel technique to eliminate potential coronal discolouration: a case report. Endod J. 2009;42(1):84-92.

29. Marcato RA, Martins LP, Prescinotti R, Cordeiro $\mathrm{RF}$, Martins TH. Agregrado trióxido mineral (mta): composição, características e relato de caso. Rev. odontol. UNESP. 2012; 41 (Spec 2).

30. Bansal R, Bansal R. Regenerative endodontics: a state of the art. Indian J Dent Res. $2011 ; 22(1): 122-31$.

31. Huang GT. Pulp and dentin tissue engineering and regeneration: current progress. Regen Med. 2009;4(5):697-707.

32. Jung $C$, Kim S, Sun T, Cho YB, Song M. Pulpdentin regeneration: current approaches and challenges. J Tissue Eng. 2019; 10: 2041731418819263.

33. Torabinejad M, Turman M. Revitalization of tooth with necrotic pulp and open Apex by using platelet-rich plasma: a case report. J Endod 2011;37(2):265-68. 


\section{CONFLITO DE INTERESSES}

Os autores declaram não haver conflitos de interesse

AUTOR PARA CORRESPONDÊNCIA

Jeane de Fátima Correia Silva Alves

Curso de Odontologia

Centro Universitário Newton Paiva

30431-262 Belo Horizonte -MG, Brasil

Email: jeane.alves@newtonpaiva.br

Submetido em 27/11/2019

Aceito em 22/10/2020 